\title{
Optimizing HVDC Grid Expansion and Control for Enhancing DC Stability
}

\section{Conference Paper}

Author(s):

Elahidoost, Atousa; Furieri, Luca (D); Tedeschi, Elisabetta; Kamgarpour, Maryam (1)

Publication date:

2018

Permanent link:

https://doi.org/10.3929/ethz-b-000292611

Rights / license:

In Copyright - Non-Commercial Use Permitted

Originally published in:

https://doi.org/10.23919/PSCC.2018.8442753 


\section{Optimizing HVDC Grid Expansion and Control for Enhancing DC Stability}

\author{
Atousa Elahidoost \\ Elisabetta Tedeschi \\ Department of Electric Power Engineering \\ Norwegian University of Science and Technology (NTNU) \\ Trondheim, Norway \\ [atousa.elahidoost, elisabetta.tedeschi]@ntnu.no
}

\author{
Luca Furieri \\ Maryam Kamgarpour \\ Automatic Control Laboratory \\ Swiss Federal Institute of Technology (ETH) \\ Zurich, Switzerland \\ [furieril,mkamgar]@control.ee.ethz.ch
}

\begin{abstract}
This paper analyzes the problem of the expansion of an offshore HVDC network. We assess which new HVDC link deployment ensures the highest stabilization of the DC voltage under transient conditions, in the worst-case scenario and for a given grid topology. A linear feedback controller is also designed to guarantee the minimization of the DC grid voltage deviations, while ensuring input constraints. The operation of this controller is compared to that of a standard droop regulator commonly applied in multiterminal Voltage Source Converter (VSC)-based HVDC networks.
\end{abstract}

Index terms-HVDC, expansion planning, linear matrix inequalities, transient stability

\section{INTRODUCTION}

The increasing growth in the number of installed offshore wind farms and the need to convey consistent amounts of intermittent wind power to load centers onshore have led to the deployment of the first point-to-point HVDC link in the North Sea [1]. The installed offshore wind capacity is expected to rise from $15.8 \mathrm{GW}$ in 2017 to $70 \mathrm{GW}$ by 2030 in Europe [2]. This anticipates the development of a multi-terminal, and eventually meshed, HVDC grid in the same area, which will emerge from the gradual interconnection of pre-existing and independently designed HVDC links. The design, control and operation of such a system will bring unprecedented challenges and opportunities.

The goal of this paper is to offer a framework based on optimal control to enhance DC voltage stability in grid expansion decisions.

Considering the technical and financial implications of deploying highly-interconnected offshore grids, the expansion planning stage is of paramount importance. However, many commonly adopted expansion planning approaches are based on techno-economical optimization and do not take stability and performance of the grid dynamics into sufficient consideration [3]. HVDC links however contribute significantly to the latter aspects and are often used to solve grid problems such as congestions and mitigation of power imbalances [4]. Their contribution with this respect should be properly accounted for when planning for a grid expansion [5].
Because of the very high cost for operation and maintenance, ensuring stability of the grid is the primary concern, especially in the offshore environment. In particular, when operating multi-terminal or meshed HVDC systems a main focus is on the DC voltage stability, which is sensitive to power imbalances due to varied wind power injection, fault conditions on the connected AC terminals and so on. [6]. The DC voltage stability can be jeopardized by resonances triggered under specific modes of operation [7]. Hence, it is necessary to ensure that the DC voltage is always kept within the allowed range at all terminals and under all scenarios of operation.

This paper provides a method to assess the stabilizing effect of the installation of a new HVDC link on the surrounding DC grid during the planning phase. Our contributions are as follows. First, for a given offshore grid topology, we assess which additional HVDC link should be installed to minimize the DC voltage oscillations, assuming the worst-case scenario for the perturbations. Second, we compute the optimal linear controller achieving such minimization. Third, we include a comparison between the proposed optimal linear controller and the droop regulator, with the goal of gaining more insight into the structure of droop regulators and the features of optimal linear controller.

Although applied to a 4-terminal test grid, the proposed optimization approach is scalable to HVDC networks of higher topological complexity in a straightforward way.

This paper is structured as follows: in Section II we present the selected test-case. In Section III we introduce the mathematical formulation of the problem using Linear Matrix Inequality theory. In Section IV we show the results obtained in terms of HVDC voltage stabilization for four different configurations and their corresponding multi-objective evaluation, and we provide a sensitivity analysis in Section V. In Section VI we present the linear feedback controller ensuring highest DC voltage stabilization and its comparison with standard droop solutions.

\section{CONSIDERED TEST CASE}

For the sake of simplicity, the approach used in this paper is exemplified using a test case based on a 4-terminal HVDC grid 


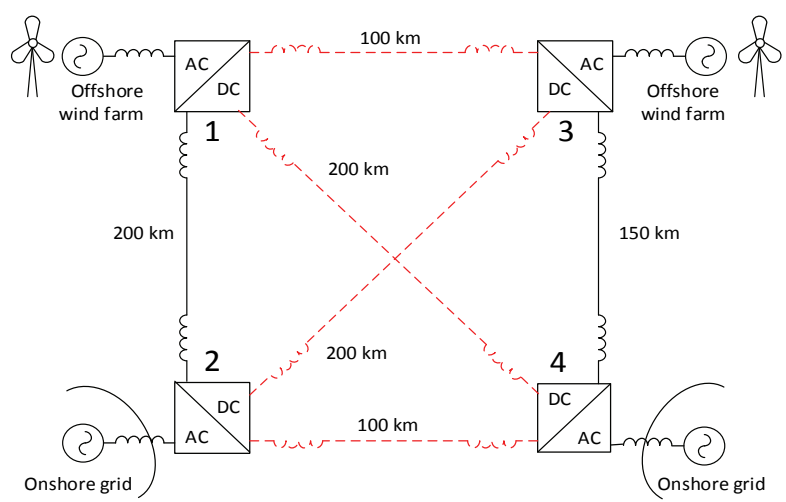

Fig. 1 4-terminal HVDC test grid [8]: existing links (solid lines) and possible expansions considered (dashed lines)

configuration [8], which is shown in Fig. 1. The system parameters correspond to those of [8] and are reported in Table I. We assume the presence of two independently operated HVDC links connecting two offshore wind farms with their respective onshore substations. The links are represented in Figure 1 by the cables between converters $1-2$ and 3-4 respectively. Assuming that we are given the possibility to extend the HVDC system with one additional link, we aim to assess how the different options, i.e. the candidate new lines 1$3,1-4,2-3$ and 2-4, would affect the resulting dynamics of the system. In order to decide on the most suitable expansion, we will exploit both the above evaluation/criterion and the length of the additional link. The HVDC converters considered in this analysis are three-phase 2-level Voltage Source Converters (2L-VSC), whose basic structure is shown in Fig. 2. The VSC converter models are developed in the synchronous reference frame [9] and the entire system is represented in per-unit (see Appendix).

We select a specific operating point, which we consider to be the most representative for the system. The chosen operating condition corresponds to specific current references for the different terminals, as indicated in Table II. The proposed analysis is based on a linearized model of each grid configuration around such equilibrium point. This equilibrium point is assumed to be the same for all the grid configurations. Thus, the system can be represented through the state-space equations $(1)-(2)$ :

TABLE I. CONVERTER AND GRID PARAMETERS OF THE SELECTED 4TERMINAL HVDC TEST GRID [8]

\begin{tabular}{|l|c|c|}
\hline & Converter $\mathbf{1 , 2 , 3}$ & Converter 4 \\
\hline Rated power [MVA] & 900 & 1200 \\
\hline AC grid voltage $[\mathbf{k V}]$ & 400 & 400 \\
\hline AC grid reactance $[\mathbf{\Omega}]$ & 17.7 & 13.4 \\
\hline AC grid resistance $[\boldsymbol{\Omega}]$ & 1.77 & 1.34 \\
\hline & Cables 1-2, 1-3, 1-4, 2-3, 2-4, 3-4 \\
\hline DC cable capacitance $[\mathbf{F} / \mathbf{k m}]$ & \multicolumn{2}{|c|}{$0.16 \mathrm{e}-6$} \\
\hline DC cable inductance $[\mathbf{H} / \mathbf{k m}]$ & \multicolumn{2}{|c|}{$0.19 \mathrm{e}-3$} \\
\hline DC cable resistance $[\mathbf{\Omega} / \mathbf{k m}]$ & \multicolumn{2}{|c|}{0.0141} \\
\hline
\end{tabular}

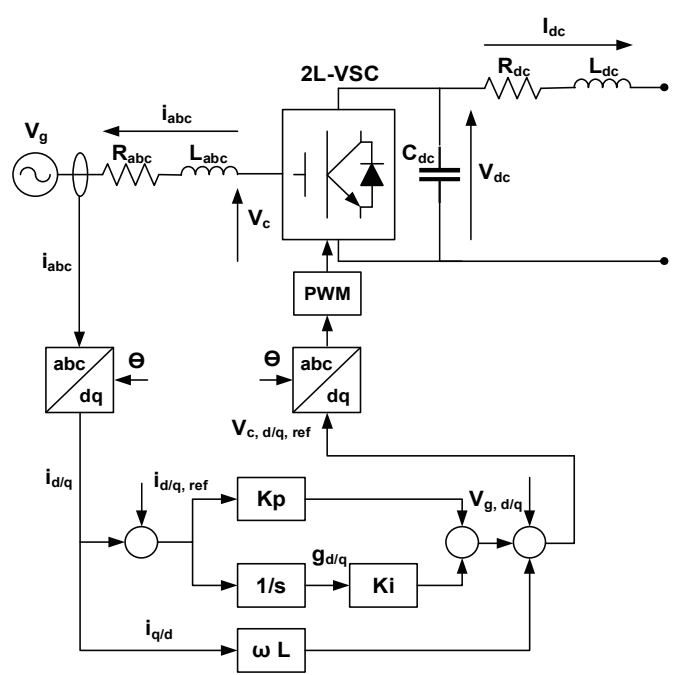

Fig. 2 Simplified model of the considered 2L-VSC

$$
\begin{aligned}
& \Delta \dot{x}(t)=A \Delta x(t)+B \Delta u(t) \\
& \Delta \mathrm{z}(t)=C \Delta x(t) .
\end{aligned}
$$

The state vector, $\Delta x(t)$, includes the variation of the state variables corresponding to all the converter stations (i.e. $d$ and $q$ components of the AC-side current and the DC voltage) and the current in the DC cables linking them.

We also assume that each converter model includes a PI current controller developed in the same $d q$ synchronous reference frame as the converter model (Figure 2). This controller, which represents the inner loop of a cascaded converter control approach, is here considered as part of the linearized system, implying the presence of two additional state variables (i.e. the controller current integral states on the $d q$ axes, $g_{d}$ and $\left.g_{q}\right)$ per converter terminal $(n=1 \ldots 4)$. The state vector corresponding to the configuration with link 1-4 is reported in ${ }^{1}$ as an example. If another configuration were studied, the last variable in the state vector would change accordingly since it represents the DC current variation of the added link.

The output vector $\Delta z(t)$ contains the signals of interest, which are in our case the DC voltage variations at the four terminals, representing a subset of the state variables.

$$
\Delta \mathrm{z}(t)=\left[\Delta v_{d c 1}, \Delta v_{d c 2}, \Delta v_{d c 3}, \Delta v_{d c 4}\right] \text {. }
$$

The input vector, $\Delta u(t)$ is composed of the reference current signal variations used to control each of the terminal converters, i.e.:

$$
\begin{aligned}
\Delta u(t)= & {\left[\Delta i_{d, r e f, 1}, \Delta i_{q, r e f, 1}, \Delta i_{d, r e f, 2}, \Delta i_{q, r e f, 2},\right.} \\
& \left.\Delta i_{d, r e f, 3}, \Delta i_{q, r e f, 3}, \Delta i_{d, r e f .4}, \Delta i_{q, r e f, 4}\right] .
\end{aligned}
$$

The consistency of the operating point is ensured by the operation of terminal 4 as a slack-bus.

1

$\Delta x_{1-4}=\left[\Delta i_{d, 1}, \Delta i_{q, 1}, \Delta v_{d c, 1}, \Delta g_{d, 1}, \Delta g_{q, 1}, \Delta i_{d, 2}, \Delta i_{q, 2}, \Delta v_{d c, 2}, \Delta g_{d, 2}, \Delta g_{q, 2}, \Delta i_{d c, 12}, \Delta i_{d, 3}, \Delta i_{q, 3}, \Delta v_{d c, 3}, \Delta g_{d, 3}, \Delta g_{q, 3}, \Delta i_{d, 4}, \Delta i_{q, 4}, \Delta v_{d c, 4}, \Delta g_{d, 4}, \Delta g_{q, 4}, \Delta i_{d c, 34}, \Delta i_{d c, 14}\right]$ 
TABLE II. SELECTED OPERATING POINT FOR THE CONVERTERS IN THE 4-TERMINAL HVDC TEST GRID

\begin{tabular}{|c|c|c|c|}
\hline $\begin{array}{c}\text { AC reference } \\
\text { current }\end{array}$ & $\begin{array}{c}\text { Reference } \\
\text { value } \\
\text { (in p.u.) }\end{array}$ & $\begin{array}{c}\text { AC reference } \\
\text { current }\end{array}$ & $\begin{array}{c}\text { Reference } \\
\text { value } \\
\text { (in p.u.) }\end{array}$ \\
\hline$I_{d, r e f, 1}$ & -0.5 & $I_{d, r e f, 3}$ & -0.7 \\
\hline$I_{q, \text { ref. } 1}$ & 0 & $I_{q, r e f, 3}$ & 0 \\
\hline$I_{d, r e f, 2}$ & 0.3 & $I_{d, r e f, 4}$ & 0.9 \\
\hline$I_{q, \text { ref }, 2}$ & 0 & $I_{q, r e f, 4}$ & 0 \\
\hline
\end{tabular}

\section{MATHEMATICAL PROBLEM FORMULATION}

In the considered analysis, we assumed that a disturbance has caused the deviation of the initial state from the nominal operating condition and we want to assess the capability of the HVDC system to counteract this deviation, in particular by minimizing the DC voltage oscillations. We need to define a performance indicator able to quantify the contribution of any additional link to the stabilization of the DC voltage of the overall multi-terminal grid. The technique we use is based on the derivations in [10], [11], which exploit the Linear Matrix Inequality (LMI) theory to determine the best HVDC link placement, with the goal of minimizing the rotor oscillations of the generators in the interconnected $\mathrm{AC}$ grid. In this paper, we use a similar approach, but the scope differs from AC grid reinforcement. For a large and highly interconnected offshore HVDC network, a higher priority is given to minimizing the variations of the DC grid voltage, in the event of external disturbances.

The selected mathematical formulation addresses the physical constraints of the system. In particular, the input is subject to ellipsoidal constraints (6), which bound the squared sum of the $d$ and $q$ reference current components, thus reflecting the maximum AC current limitation of each converter. To this end, we correspondingly divide $\Delta u$ as

$\Delta u=\left[\begin{array}{llll}\Delta u_{1}^{T} & \Delta u_{2}^{T} & \Delta u_{3}^{T} & \Delta u_{4}^{T}\end{array}\right]^{T}$,

where $\Delta u_{n} \in R_{2}, \forall n=1 \ldots 4$ and it must satisfy:

$\Delta u(t) \in \mathrm{U}=\left\{\Delta u \in R_{8}: \Delta u_{n}^{T} E_{u, n} \Delta u_{n} \leq 1, \forall n=1 \ldots 4\right\}$,

$E_{u, n} \in R_{2 x 2}$ being a symmetric positive definite matrix.

In a similar way, the initial state $\Delta x(0)$ is assumed to lie within an ellipsoidal set $X_{0}$, which represents the set of possible worst-case conditions assumed by the system after any perturbation:

$\Delta x(0) \in X_{0}=\left\{\Delta x \in R_{23}: \Delta x^{T} E_{x} \Delta x \leq 1\right\}$,

$E_{x}>0$ being a symmetric positive definite matrix.

The performance indicator we select to quantify the degree of stability of the DC voltage against disturbances is expressed as the time-integral of a quadratic function of the DC voltage variations vector, that is:

$J_{\text {perf }}=\int_{0}^{\infty} z(t)^{T} M z(t) d t$

where the matrix $M$ is positive definite $(M>0)$ and acts as a weight on the signals of interest.

The goal of our study is to identify, among a set of possible grid topologies, the one that minimizes the disturbance- induced oscillations on the DC voltage under the worst-case scenario, with the action of an appropriate controller. We consider the use of a linear feedback in order to design this DC stabilizing controller:

$u(t)=K x(\mathrm{t})$.

As mentioned, $u(t)$ represents the outer controller of a cascaded feedback loop, and it provides the current references to the inner current regulator that is based on standard PI controllers whose dynamics are included in our system of equations (1) and (2). Additionally, the proposed analysis allows identifying the optimal controller $K$ ensuring the best performance.

\section{IDENTIFICATION OF THE OPTIMAL EXPANSION OPTION}

The choice of the optimal feedback gain, $K$, in (9) with the highest stabilizing effect on a selected grid configuration requires the solution of the following min-max optimization problem (10):

$J_{\text {perf }}^{*}=\min _{K} \max _{x(0) \in X_{0}} J_{\text {perf }}$

s.t. (1), (2), (6), (7), (8), (9).

The direct computation of the above is difficult. However, under the assumption of ellipsoidal bound for input constraints (6) and initial state (7), and with the choice of a quadratic performance indicator (8), we can formulate the problem using Linear Matrix Inequalities, as shown in [10], [11]:

$\frac{1}{J_{p e r f}^{*}}=\max _{s>0, Q>0, Y} s$

s.t.

$0 \leq\left[\begin{array}{cc}(A Q+B Y)+(A Q+B Y)^{T} & Q C^{T} \hat{M} \\ \hat{M}^{T} C Q & -I\end{array}\right]$

$0 \leq Q-s E_{x}^{-1}$

$0 \leq\left[\begin{array}{cc}Q & Y_{n}^{T} \\ Y_{n} & s E_{u, n}^{-1}\end{array}\right], \forall n=1 \ldots 4$

where $Q \in R_{23 \times 23}, \quad Y_{n} \in R_{2 \times 23} \forall n=1 \ldots 4, \quad Y=\left[\begin{array}{llll}Y_{1}^{T} & Y_{2}^{T} & Y_{3}^{T} & Y_{4}^{T}\end{array}\right]^{T}$ and $s$ is a scalar quantity whose inverse represents the upper bound of $J_{\text {perf }}$ and $\hat{M}$ is derived from the factorization of $M=\hat{M} \hat{M}^{T}$. Once the optimal solution $\left(S^{*}, Q^{*}, Y^{*}\right)$ is obtained, it is possible to recover the optimal feedback gain for the worst initial condition as $K_{w}^{*}=Y^{*} Q^{*}$ and apply the corresponding optimal control input $u^{*}(t)=K_{w}^{*} x(t)$. Additionally, it is possible to compute the worst case initial oscillation $\Delta x_{w}(0)$ as $\Delta x_{w}(0)=\hat{E}^{-T} v_{1}$, where $\hat{E}=V D^{1 / 2}, \quad V$ contains the eigenvector of $E_{x}, D$ is diagonal with the eigenvalues of $E_{x}$ and $v_{l}$ is the eigenvector of $\hat{E}^{-1} Q^{*-1} \hat{E}^{-T}$ associated with its largest eigenvalue.

The matrices $A, B$ and $C$, correspond to the state space matrices in (1) and (2) and they will therefore differ for each of the four grid configurations considered. The matrix $M$ is selected here as the unity matrix. 


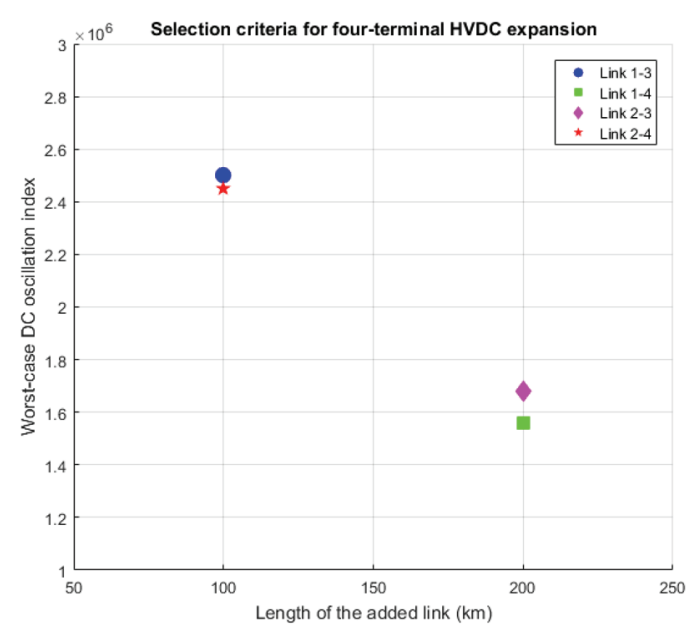

Fig. 3 Comparison of the considered expansion options

The matrices $E_{u, n}$, are selected to be diagonal matrices enforcing (6) with the actual current constraints of each terminal. This assumes the total current to be bounded to take into account the limits on the maximum possible overcurrent that the converter switches can withstand, when operating close to nominal power, i.e.:

$$
\left|\Delta i_{r e f, n}\right|=\sqrt{\Delta i_{d, r e f, n}^{2}+\Delta i_{q, r e f, n}^{2}} \leq 0.25 p . u \text {. }
$$

The matrix $E_{x}$ is also a diagonal matrix, that enforces constraints (7), on maximum assumed deviations of the initial state variables (13-16). The AC current limitations reflect those imposed to the corresponding references in (7), and the DC voltage and DC current ones are associated with physical limitations of the DC cable.

$$
\begin{aligned}
& \left|\Delta i_{n}\right|=\sqrt{\Delta i_{d, n}^{2}+\Delta i_{q, n}^{2}} \leq 0.25 \text { p.u. } \\
& \left|\Delta V_{d c, n}\right| \leq 0.1 p . u . \\
& \left|\Delta g_{n}\right|=\sqrt{\Delta g_{d, n}^{2}+\Delta g_{q, n}^{2}} \leq 0.2 p . u . \\
& \left|\Delta J_{d c, n}\right| \leq 0.1 \text { p.u. }
\end{aligned}
$$

The maximization problem (11) has been solved using the Yalmip toolbox [12] of Matlab ${ }^{\circledR}$ to determine $J_{\text {perf }}^{*}$ for all the configurations. In the considered test case, the four possible expansion options are assessed and compared based on the twofold criteria of: a) minimum cable length; and b) minimum DC voltage oscillations (i.e. minimum $J_{p e r f}$ ). Assuming that an equal weight is given to both objectives, represented on orthogonal axes in Fig.3, we can see that link 1-4 would be the preferable option, as it represents the point at minimum distance from the origin. However, it is possible to allocate different weights to each criteria based on planning phase preferences and priorities, which may result in the final selection of a different preferable configuration.

From the results obtained for the four configurations, we can notice that the $J_{\text {perf }}^{*}$ index is highly dependent on the length of the added HVDC link. The higher the cable length, the lower the DC oscillation index and the better the performance under worst-case initial disturbances. This implies that the two selected objectives are conflicting. This result is, however, physically sound and can be explained by the fact that an increase of the line length, and consequently of the line capacitance, results in DC voltage ripple reduction and stability enhancement. Moreover, the better performance of links 1-4 and 2-4 compared to links 1-3 and 2-3, can be due to the operation of terminal 4 as a slack bus compensating for the network power balance while its voltage is kept constant at the reference value. The numerical results provide quantitative measure for the explanation above.

\section{Parameter Sensitivity OF THE OPtimal CONFIGURATION}

We have performed a parameter sensitivity analysis, by halving and doubling the cable equivalent kilometric capacitance, inductance and resistance (alternatively), compared to the base case, to analyze the dependence of the expansion decision on the line parameters and further confirm the physical explanation of the voltage stabilization previously proposed. From Fig. 4, we can clearly see that the cable capacitance has the biggest influence on the $J_{\text {perf }}^{*}$ index, with larger deviations in the behavior of links of equal length obtained for lower capacitances. On the contrary, and according to intuition, resistors and inductors do not have significant effect on the voltage stabilization. The analysis
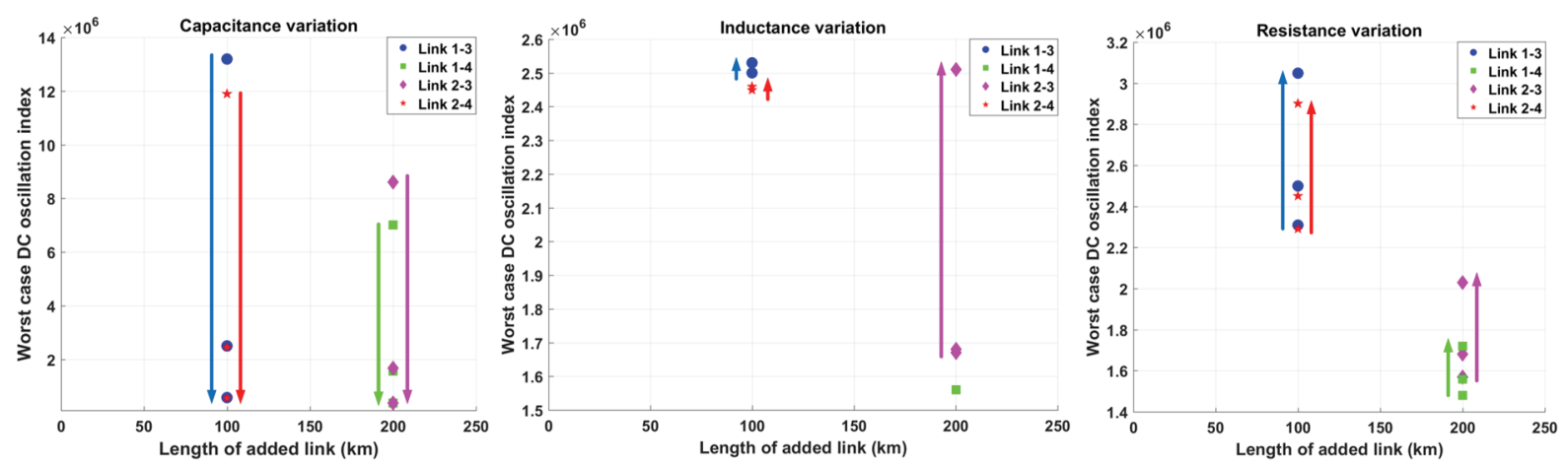

Fig. 4 Comparison of the considered expansion options based on cable parameters' sensitivity analysis: a) capacitor variation, $0.08,0.16 \& 0.32 \mathrm{uF} / \mathrm{km}$ (left), b) inductor variation $0.095,0.19 \& 0.38 \mathrm{mH} / \mathrm{km}$ (middle), c) resistance variation $0.007,0.014 \& 0.028 \Omega / \mathrm{km}$ (right) (Arrow direction shows the parameter's value increase.) 

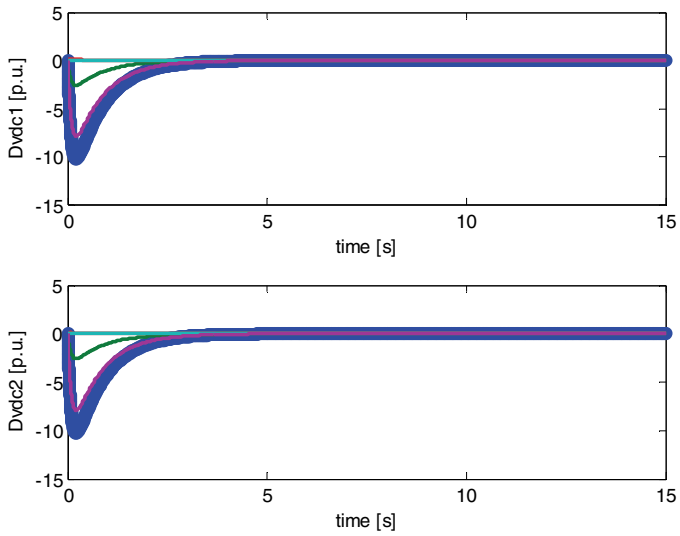
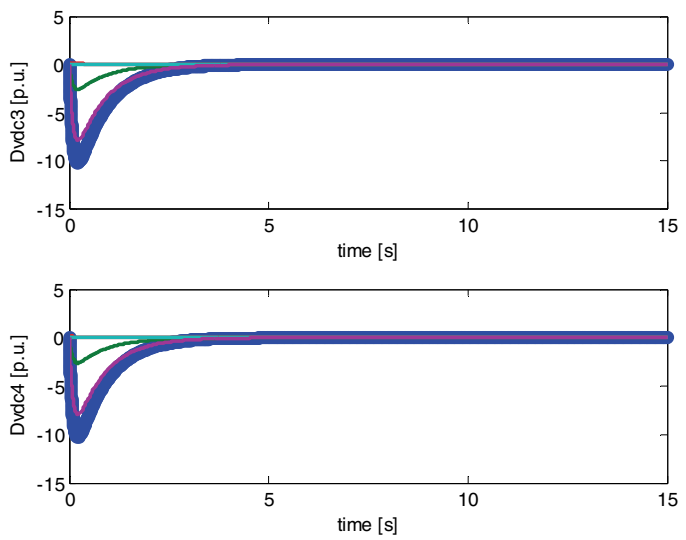

Fig. 5 System response of the DC voltage at terminal 1 (top left), terminal 2 (bottom left) terminal 3 (top right) and terminal 4 (bottom right). In all graphs, the blue trace corresponds to DC voltage evolution starting from the identified "worst-case" initial conditions, and the other curves correspond to other randomly selected initial conditions within the allowed range

shows that even considering wide ranges of variation for the cable kilometric parameters, the line characteristic having the highest impact on the reduction of DC voltage oscillation is the cable length. Even though the $J_{\text {perf }}^{*}$ values vary due to cable parameters' variation, the comparison among the four grid configurations continues to indicate link $1-4$ as the optimal one.

\section{Optimal CONTROLleR For MaXimum DC Voltage STABIILITY}

The second result of the proposed study is the mathematical derivation of the linear feedback controller that allows achieving the maximum stabilization of the system voltage in the worst-case scenario.

As explained in the previous section, the algorithm allows identifying the worst-case initial condition $\Delta x_{w}(0)$, within the allowed region described by (7) and (12-16), which produces the highest oscillation in the DC voltages, and targets the optimal design of the controller $K$ to that specific initial condition.

In the considered configurations, the identified worst case condition corresponds to a vector $\Delta x_{w}(0)$ representing the maximum deviations of the $d$-component of the current integral state for each of the converters $\Delta g_{d, n}$. It is interesting to note the higher sensitivity of the DC voltage stabilization index to a control variable (i.e. the integral of the state corresponding to the d-current) than to physical parameters depending on the network configuration.

Fig. 5 shows the free system response (i.e. the DC voltages of the terminals), starting from the worst-case initial deviation identified by the algorithm (thicker line), and four other randomly selected realistic initial conditions. It can be seen how higher DC voltage oscillations with respect to other random conditions are detected in the identified worst-case.

In order to take a closer look at the optimal controller structure, the controller obtained for the configuration including link 1-4 is reported in ${ }^{2}$, as an example. Controllers derived for the other grid configurations have similar structure.

Even rows correspond to the $q$-components of the reference currents, and, as expected, they are all equal to zero, since DC voltage oscillations indicate active power imbalances and hence should be associated to an action on $d$-current components.

Note that the linear feedback acts on the states corresponding to the DC voltage and $d$-current integrals of all the four terminals, as well as on the $d$-current of terminals 1,3 and 4 . This can be explained since 1 and 3 are directly connected terminals in this configuration and terminal 4 acts as the slack bus.

It is also worth noting that the optimal controller we computed is sparse. However, it is centralized, in the sense that each terminal requires some information from all the other terminals in order to synthesize the current references.

2

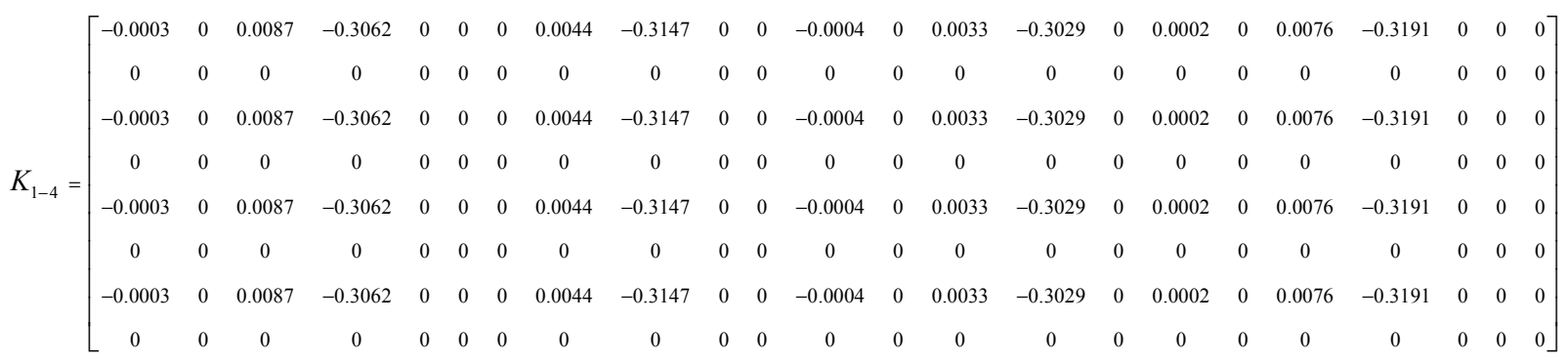




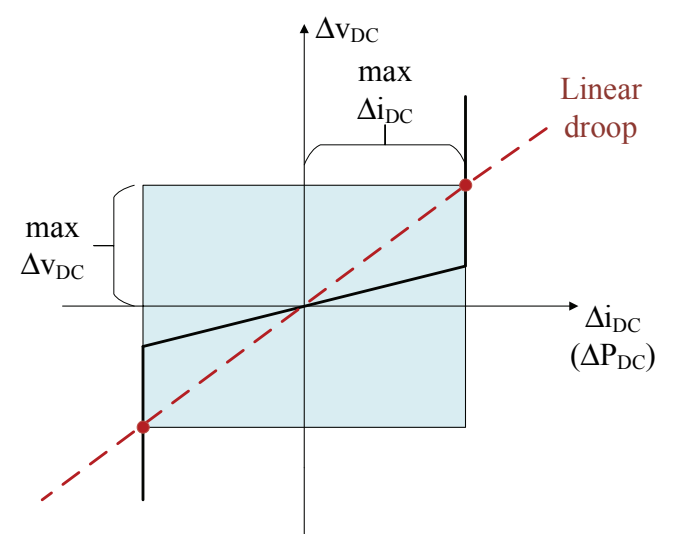

Fig. 6 Non-linear droop controller (black line) and its linear approximation (red dashed line) complying with the same $\Delta \mathrm{i}_{\mathrm{DC}}$ and $\Delta \mathrm{v}_{\mathrm{DC}}$ limits

On the other hand, a classic droop controller, which is often used as the outer loop controller in such HVDC applications, can also be represented via linear feedback in normal operating conditions. A power feedback and a current feedback, both expressed in the per-unit system, can be considered equivalent provided that the voltage is considered almost constant.

In the case of a droop controller, however, the structure of the matrix $K$ would be much simpler. The controller would be decentralized and would have only four non-zero elements. They would be found in odd rows, on the columns corresponding to the DC-voltage at the same terminal of the $d$ current reference considered.

However, the design of a droop controller is often based on experience rather than on analytical procedures and the droop coefficients, $\Delta \mathrm{v}_{\mathrm{DC}} / \Delta \mathrm{P}_{\mathrm{DC}}$, normally used are in the range $2-10 \%$. Considering the maximum allowed DC voltage oscillation of our case, such limited droop would imply the need of significant $I_{D C}$ current injections by the terminals, which may, in turn, conflict with our considered DC current limitation in (16). In practical applications, droop controllers normally take into account the physical system limitations (i.e. current or power limits) by inserting proper saturations on the controlled signals (Fig. 6). This makes the representation highly nonlinear and difficult to capture by the linearized approach proposed. A linearized representation of the droop action compatible with the problem formulation of Section III, and compliant with the state constraints set in our optimization algorithm (7), encompassing the maximum deviation on both $v_{D C}$ and $i_{D C}$ (red points in Fig. 6) requires a higher droop slope than the actual droop coefficient used in realistic cases. Thus, due to the non-linear nature of the real droop controller a quantitative comparison with the proposed controller is not straightforward. However, based on additional tests, a qualitative comparison between the optimal controller derived analytically and the traditional droop controller is presented in Table III.

The possibility of analytically identifying the worst-case DC voltage oscillations and designing a suitable stabilizing controller, without the need of dynamic models and multiple simulations can be especially useful at the preliminary stage of expansion planning, when a multitude of parameters and
TABLE III: QUALITATIVE COMPARISON BETWEEN THE PROPOSED OPTIMAL CONTROLLER AND STANDARD DROOP CONTROLLER

\begin{tabular}{|l|l|}
\hline $\begin{array}{l}\text { Optimal linear feedback } \\
\text { controller }\end{array}$ & Standard droop controller \\
\hline $\begin{array}{l}\text { Is obtained by analytical } \\
\text { derivation considering system } \\
\text { physical constraints }\end{array}$ & $\begin{array}{l}\text { Requires case-by-case tuning } \\
\text { with ex-post check on } \\
\text { constraints compliance }\end{array}$ \\
\hline $\begin{array}{l}\text { Ensures best performance under } \\
\text { the worst case scenario }\end{array}$ & $\begin{array}{l}\text { May provide suboptimal } \\
\text { performance in the worst case } \\
\text { scenario }\end{array}$ \\
\hline $\begin{array}{l}\text { When a specific scenario is } \\
\text { considered, it can be used to } \\
\text { ensure the best performance } \\
\text { under such scenario }\end{array}$ & $\begin{array}{l}\text { The same droop controller can } \\
\text { ensure good performances in } \\
\text { most cases }\end{array}$ \\
\hline Centralized solution & Decentralized solution \\
\hline
\end{tabular}

scenarios should be considered and detailed simulations of all of them is prohibitive. In this way, the stability enhancement contribution can be easily included as an additional parameter in a multi-objective optimization, before deciding which link to deploy. Once the grid decision is made, we can implement decentralized droop controllers, having simpler structure, independence on long-distance communication, and good performance in a plurality of realistic conditions.

As an additional point, it is interesting to highlight the high sensitivity of the DC voltage stabilization index to the current integral state variable variation $\Delta g_{d, n}$.

This is as an additional reason to support the need for an integrated analysis between control/stability aspects, and expansion planning studies. In fact, enhanced stability and controllability should be given an economic value when the deployments of new HVDC links is considered.

\section{CONCLUSIONS}

We investigated the problem of assessing different expansion options in offshore multi-terminal HVDC grids. A performance index that is capable to quantify how each added DC link can contribute to the reduction of DC voltage oscillations in the system is introduced. Such index can be used as one of the decision criteria in expansion planning, in conjunction with other technical and economic metrics.

Few previous contributions included control and stability aspects in the assessment of HVDC expansion options. Unlike them, our paper has a specific focus on large off-shore systems, where the DC voltage stabilization has a higher priority than oscillation damping in the interconnected AC grids.

Moreover, we have presented a mathematical formulation that allows decoupling the boundaries of the input signals of one terminal from those of the other terminals in the grid, thus ensuring more fidelity to the real implementation than previous similar works. We have exemplified the proposed approach using a 4-terminal HVDC test grid. Nevertheless, this can be easily extended to hybrid AC/DC systems and more complex (e.g. meshed) configurations, by simply modifying the linearized dynamical system matrices accordingly.

We have discussed the merits of the proposed approach in the context of HVDC expansion planning, and analyzed the characteristics of the proposed optimal controller versus a standard droop controller, normally used for such applications. 
We plan to investigate decentralized optimal control as part of our future work, with the goal of retaining the advantages of both the optimal linear feedback controller and traditional droop controllers.

\section{ACKNOWLEDGEMENT}

Authors A. Elahidoost and E. Tedeschi are working under the project of "Integrated Design and Control of Offshore HVDC Networks (IDeCON)" financially supported by the Norwegian Research Council and DNV GL. The work of M. Kamgarpour and L. Furieri is gratefully supported by ERC Starting Grant CONENE.

\section{REFERENCES}

[1] ABB Reference projects, "BorWin1: One of the most remote offshore wind farm clusters in the world is connected to the German grid by a 400 MW +/-150 kV HVDC Light transmission system from ABB," [online]. Available: http://new.abb.com/systems/hvdc/references/borwin1

[2] Wind Europe, "Wind in power 2017" February 2018, [online]. Available: https://windeurope.org/about-wind/statistics/european/windin-power-2017/

[3] A. Elahidoost and E. Tedeschi, "Expansion of offshore HVDC grids: An overview of contributions, status, challenges and perspectives," in 2017 IEEE 58th International Scientific Conference on Power and Electrical Engineering of Riga Technical University (RTUCON), 2017, pp. 1-7.

[4] A. A. van der Meer, M. Ndreko, J. A. Bos, M. Gibescu, M. A. M. M. van der Meijden and W. L. Kling, "Stability assessment of VSC-HVDC connected large-scale offshore wind power: A North-Sea region case study," 2015 IEEE Eindhoven PowerTech, Eindhoven, 2015, pp. 1-6.

[5] M. Henderson, J. Gagnon and D. Bertagnolli, "Planning issues for HVDC," 2006 IEEE PES Power Systems Conference and Exposition, Atlanta, GA, 2006, pp. 28-32.

[6] Wang, W., Barnes, M., Marjanovic, O., \& Cwikowski, O. (2016). Impact of DC breaker systems on multiterminal VSC-HVDC stability. IEEE Transactions on Power Delivery, 31(2), 769-779.

[7] G. Pinares "Analysis of the DC dynamics of VSC-HVDC systems connected to weak AC grids using a frequency domain approach" Power Systems Computation Conference (PSCC), 2014, Wroclaw, Poland, p17.

[8] W. Leterme, N. Ahmed, J. Beerten, L. Angquist, D.V. Hertem, S. Norrga, "A new HVDC grid test system for HVDC grid dynamics and protection studies in emt-type software," 11th IET International Conference on AC and DC Power Transmission, pp. 1-7, Feb 2015

[9] S. Cole, J. Beerten and R. Belmans, "Generalized Dynamic VSC MTDC Model for Power System Stability Studies," in IEEE Transactions on Power Systems, vol. 25, no. 3, pp. 1655-1662, Aug. 2010.

[10] A. Fuchs and M. Morari, "Actuator performance evaluation using LMIs for optimal HVDC placement," ECC Conference, Zurich 2013, pp. 152934.

[11] A. Fuchs, and M. Morari "Placement of HVDC links for power grid stabilization during transients," IEEE PowerTech, 2013 IEEE Grenoble.

[12] J. Löfberg, "Yalmip: A toolbox for modeling and optimization in MATLAB," Proceedings of the CACSD, Taipei, Taiwan, 2004.

\section{APPENDIX}

BASE VALUES USED FOR THE P.U. SYSTEM

Base power, $S_{b}$ :

1200 MVA

AC base voltage, $V_{A C, b}$ :

$\sqrt{2 / 3} \times 400 \mathrm{kV}$

DC base voltage, $V_{D C, b}$ :

$$
2 \times \sqrt{2 / 3} \times 400 \mathrm{kV}
$$

